\title{
A decision modelling approach for selection of biological nutrient removal systems for wastewater
}

\author{
Carla Mae Pausta ${ }^{1}$, Ramon Christian Eusebio ${ }^{2}$, Arnel Beltran ${ }^{1}$, Aileen Huelgas-Orbecido ${ }^{1}$, and Michael Angelo \\ Promentilla ${ }^{1, *}$ \\ ${ }^{1}$ Chemical Engineering Department, De La Salle University, Manila City \\ ${ }^{2}$ Department of Engineering Science, University of the Philippines Los Baños, Laguna
}

\begin{abstract}
This paper proposes a decision model built on a hierarchical network for optimal selection of biological nutrient removal systems (BNR) in wastewater treatment plants. BNR is an important component of a sustainable wastewater management wherein resource recovery from wastewater becomes an integral part of the municipal wastewater treatment plants (WTP). However, selection of the most appropriate technology or systems requires a multiple criteria analysis. This study focuses on the following criteria namely 1) Economic aspect; 2) Technical aspect; 3) Environmental Aspect; and 4) Space Requirement. The following alternatives were then evaluated: 1) 3 Stage Pho-redox (A2O); 2) 5 Stage Bardenpho (5BP); 3) University of Cape Town (UCT); 4) Virginia Initiative Plant; 5) Sequencing Batch Reactor (SBR); 6) Membrane Bioreactor (MBR). A fuzzy ANP approach with Monte Carlo simulation was used to derive the overall priorities of these alternatives. This decision modelling approach addresses the uncertainty and complexity involved in the selection of appropriate BNR in Metro Manila's WTP.
\end{abstract}

\section{Introduction}

Nitrogen $(\mathrm{N})$ and phosphorus $(\mathrm{P})$ are the major nutrients needed in order to sustain and improve growth of plants both in land and in water. While nutrients in the form of fertilizers create beneficial effects to agricultural land, conversely, excessive nutrient concentration in the water bodies causes adverse effects to the aquatic biodiversity. This leads to eutrophication which is the accelerated growth rate of aquatic plants and algae due to increased availability of nutrients. Algal bloom and excessive growth of aquatic plants limit light penetration and deplete dissolved oxygen which are needed to support aquatic organisms [1]. Common sources of nutrient pollution in the waters are storm water run-off, wastewater effluent and agricultural water run-off. With that, efforts are being made to address this problem by researchers and industries involved. In order to control the nutrient concentration in the wastewater effluent, different technologies were developed including biological nutrient removal (BNR) systems and nutrient recovery systems [2-4]. Several countries have already started the monitoring of nutrient content in water and wastewater discharges especially in their wastewater treatment plants (WTP) [2-3].

The domestic and industrial WTPs in the Philippines are still on the planning stages of implementation of BNR technologies. The Department of Environment and Natural Resources (DENR) released a new Department
Administrative Order (DAO) 2016-08 last May 2016 indicating the new water quality guidelines and general effluent standards starting 20162016 with the inclusion of ammonia-N (NH3-N), Nitrate-N (NO3-N) and Phosphorus as Phosphates among the monitored parameters [5]. The government gave a grace period of not more than five years to comply with the new DAO. Because of that, WTPs need to upgrade their current system that will integrate BNR technologies in order to comply with the new standards.

The challenge faced by the stakeholders is the selection of the best BNR system to be installed in municipal WTPs located in urban areas. Water and wastewater treatment systems are complex and dynamic since treatment is influenced by interactions of factors such as water quality, regulatory requirements, consumer and environmental concerns, construction challenges, operational constraints, and economic feasibility [6]. The United States Environmental Protection Agency (USEPA) identified the following barriers in the implementation of BNR system: 1) costs; 2) limitations on physical expansion; 3) state resources; 4) increased carbon footprint; 5) advanced operations and control [4]. Industries with WTPs are now faced with a complex decision making problem to evaluate the best BNR alternative considering multiple criteria.

This study will focus on the selection of the optimum BNR system using Fuzzy Analytical Network Process (FANP), a Multi-criteria Decision Analysis (MCDA)

* Corresponding author: michael.promentilla@dlsu.edu.ph 
tool, with uncertainty analysis. MCDA is a tool that can provide decision support to complex and multifaceted problems considering the interconnection of various factors [6-8]. Common MCDA methods used in different environmental decision making problems are Multiattribute Utility Theory (MAUT), Analytical Hierarchical Process (AHP) and Analytical Network Process (ANP). AHP utilizes pairwise comparisons of all criteria and alternatives to institute relationships within the hierarchical problem structure [9]. ANP is the generalization of AHP which considers the interactions and dependence between the elements of clusters or networks [10]. A study was made to evaluate the best BNR technology for WTP in urban areas using ANP [11]. However, the ambiguity in the value judgements provided by experts and uncertainties involved were not considered. In this paper, Monte Carlo simulations are done to model the variabilities caused by subjective judgements and coarse quantitative data [12].

\section{Methodology}

The study proposed a decision modelling approach utilizing the fuzzy concept in Analytical Network Process coupled with Monte Carlo simulations in order to solve the complex decision making problem as summarized in Fig. 1.

Setup the Network Structure.

Obtain the triangular fuzzy numbers through value judgements from experts and data collected from literature.

Calculate the priority vectors using the nonlinear fuzzy preference programming approach

The supermatrix containing the priority vectors will be normalized per cluster through row sum then raise to large powers until it converges to a limit.

Evaluate the uncertainties using Monte Carlo simulations.

Fig. 1. Methodology flow chart

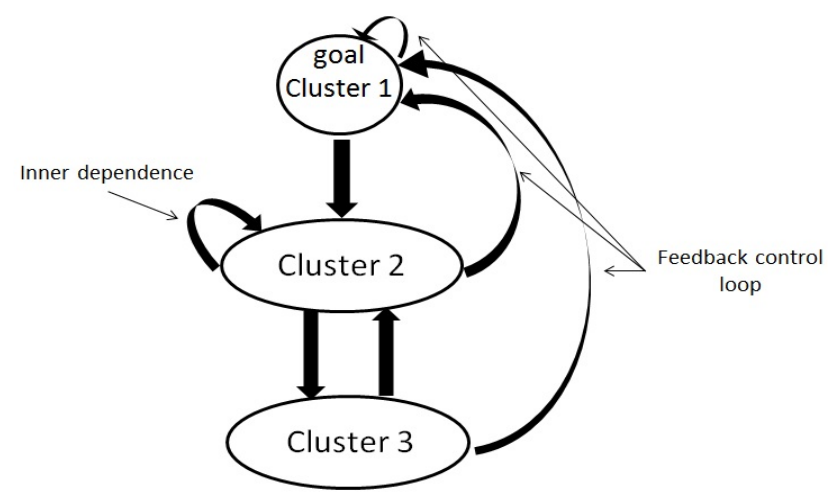

Fig. 2. An example of ANP network structure
The first phase was to setup a network structure to represent the problem as shown in Fig. 2. The network structure has a top to bottom hierarchy followed by feedback from alternatives to criteria, inner dependence of all criteria, and feedback control loop as represented by the direction of the arrows and arc. The feedback control loop indicates that all the elements in the structure are important and influenced by the goal element [13].

The second phase was to gather pairwise comparisons of all interacting elements in the network structure through value judgements from experts and data collected from literature. The relative importance between criterion $i$ and criterion $j$ is represented by a triangular fuzzy number (TFN) which is described by $<$ lower bound, modal value, upper bound $>\left(<l_{i j}, m_{i j}, u_{i j}>\right)$ to address the ambiguity of the value judgements $\left(a_{i j}\right)$ provided by the experts [12]. The lowest relative judgement provided by an expert was considered as the lower bound $\left(l_{i j}\right)$ value while the highest judgement was considered as the upper bound $\left(u_{i j}\right)$ value. The modal $\left(m_{i j}\right)$ value was obtained by calculating the geometric mean of the relative value judgments. The geometric mean method was used to get the aggregated value of the expert judgments taken from multiple decision makers [14]. The pairwise comparison matrix is presented in eq. (1).

$$
\hat{A}=\left[\begin{array}{cccc}
\langle 1,1,1\rangle & \hat{a}_{12} & \cdots & \hat{a}_{1 n} \\
\hat{a}_{21} & \langle 1,1,1\rangle & \ldots & \hat{a}_{2 n} \\
\vdots & \vdots & \ddots & \vdots \\
\hat{a}_{n 1} & \hat{a}_{n 1} & \cdots & \langle 1,1,1\rangle
\end{array}\right]
$$

$$
\text { where } \hat{a}_{j i}=\left\langle l_{i j}, m_{i j}, u_{i j}\right\rangle ; \hat{a}_{j i}=\left\langle\frac{1}{u_{i j}}, \frac{1}{m_{i j}}, \frac{1}{l_{i j}}\right\rangle
$$

The third phase involved the calculation of the priority weights using the nonlinear fuzzy preference programming approach described in the paper of Promentilla et. al. [15]. The approximate ratio-scale weights $\left(w_{i}\right)$ were obtained, wherein the value of $\lambda$ was maximized. The $\lambda$ value was used as a measure of consistency where a value of 1.0 means perfect consistency while a value of 0.0 indicates that the fuzzy judgments are only satisfied at their boundaries [16].

In the fourth phase, the normalized priority vectors were arranged in a supermatrix, $\boldsymbol{S}$, that represents the network structure of the decision making problem to calculate for the overall priority weights. The initial supermatrix or the unweighted matrix is composed of priority weights obtained from the previous step. This was normalized by the row sum. The supermatrix was then raised to large powers until it converged into a limiting value [17]. The resulting answers were normalized per cluster to get the overall priorities.

The last phase was to evaluate the uncertainty in the computation of the overall priority weights using Monte Carlo simulations [18]. This will also address the ambiguity of value judgments provided and probable imprecise quantitative data from different literatures. 


\section{Case Study}

This study evaluates the best biological nutrient removal (BNR) system to be installed in existing wastewater treatment plants (WTPs) located in the urban areas based on the following criteria: (1) economic aspect (EC); (2) technical aspect (TL); (3) environmental aspect (EN); and (4) space requirements (SR). EC considers lower upgrade costs (i.e. construction costs, etc.). The economic aspect data of alternatives to be evaluated are taken from different literatures [19-21]. The cost data are then normalized using the Engineering News Record Construction Cost Index [19]. TL considers the robustness of the processes and the technical capabilities of the assigned personnel. EN is being evaluated by the effluent quality especially but not limited to lower nitrogen and phosphorus concentration in the effluent. The effluent quality must pass the new standards set by the new DAO 2016-08 at the minimum. However, for the comparison of elements with respect to EN, priority must be given to the system with effluent having the least nutrient concentration. SR refers to the physical expansion and upgrade of conventional WTP when the BNR is integrated. In cities, addition of spaceconsuming technology such as BNR systems must also be considered as an important factor. In this criterion, the priority must be given to the alternative with lesser space requirement.

The following are the six alternatives to be evaluated: 3 stage pho-redox (A2O), 5-stage Bardenpho (5BP), University of Cape Town (UCT), Virginia Initiative Plant (VIP), Sequencing Batch Reactor (SBR) and Membrane Bioreactor (MBR). A2O is a conventional activated sludge system involving anaerobic-anoxicaerobic zone. In order to enhance denitrification, the nitrate-rich liquor is recycled from the aerobic zone to the start of the anoxic zone. The return activated sludge (RAS) is returned in the anaerobic zone. The 5BP consists of five stages with anaerobic-anoxic-aerobicanoxic-aerobic zones. The nitrate-rich liquor is recycled to the first anoxic stage from the first aerobic stage while the RAS is recycled to the beginning of the anaerobic stage from the clarifier. UCT also consists of three stages, anaerobic-anoxic-aerobic stage. However, the RAS is returned from the clarifier to the anoxic stage while the nitrate-rich liquor is recycled from the aerobic stage to the anoxic stage [4]. VIP having the same anaerobic-anoxic-aerobic stages recycles its RAS to the anoxic stage where denitrification occurs before entering the anaerobic stage [22]. SBR is a batch operation of anaerobic-anoxic-aerobic stages through adjustment of mixing and aeration [4]. MBR utilizes membranes for the nutrient removal [23].

The complex decision making problem for the optimum selection of BNR system can be summarized in a network structure shown in Fig. 3 which considers the aforementioned set of criteria and six possible alternatives. Since the selection of the BNR system is influenced by a cluster of criteria, it is represented by an arrow from the goal cluster to the criteria cluster, denoted by $W_{21}$ in the supermatrix, $\boldsymbol{S}$. However, the evaluation of these criteria may also be influenced by other criteria, thus the inner dependence represented by the arc loop must also be considered in the supermatrix as $W_{22}$. The preferred alternative with respect to each criterion, $W_{32}$, is also being assessed as represented by the arrow from the criteria cluster to the alternative cluster. On the other hand, the evaluation of each alternative may be influenced by the dominating criteria, thus the feedback is considered as represented by the arrow from the alternative cluster to the criteria cluster in the submatrix $W_{23}$. Since there are no dependencies between the alternatives, it will be assigned as a null submatrix, $W_{33}$, in the supermatrix. A null matrix is also assigned to $W_{31}$ because the goal is manifestly not dependent to the each of the alternatives. The feedback control loop is represented by the arcs pointing to the goal cluster from the goal, criteria and alternative clusters. This indicates that all the elements in the structure are influenced by the single controlling element, goal, as indicated by the value of 1.0 in the submatrices, $W_{11}, W_{12}, W_{13}$, respectively.

$$
S=\left[\begin{array}{lll}
W_{11} & W_{12} & W_{13} \\
W_{21} & W_{22} & W_{23} \\
W_{31} & W_{32} & W_{33}
\end{array}\right]
$$

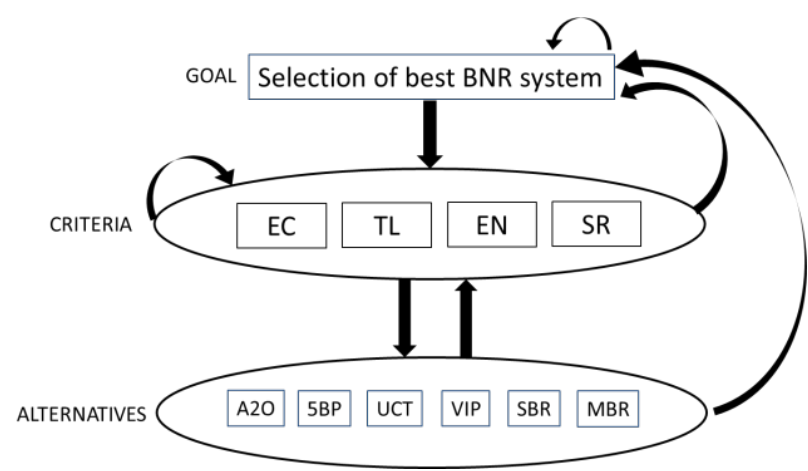

Fig. 3. ANP network structure for the selection of best BNR systems in urban areas

Three experts from the academe participated in the provision of value judgments through pairwise comparisons of the elements in the clusters. The TFN described by $\left\langle l_{i j}, m_{i j}, u_{i j}>\right.$ are composed of the lowest value judgement, geometric mean of all judgements and the highest value judgement respectively. Using the nonlinear fuzzy preference programming approach with the aid of Lingo, the approximate ratio-scale weights $\left(w_{i}\right)$ are obtained while maximizing the value of $\lambda$.

The priority vectors from the pairwise comparison matrix for all the interacting elements are populated in an unweighted supermatrix, shown in Table 1. All elements in the submatrices $W_{11}, W_{12}$ and $W_{13}$ have a value of 1.0 that can be attributed to the influence of the goal which is the single controlling element. The submatrix $W_{21}$ shows the resulting priority vectors from the pairwise comparison of the relative importance of each criterion with respect to goal. The submatrices $W_{22}$, $W_{23}$ and $W_{32}$ are populated by priority vectors considering the inner dependence of evaluation of criteria, feedback of alternatives to criteria, and evaluation of preferred of alternatives considering the 
criteria, respectively. $W_{3 l}$ is a null matrix that contains a value of 0.0 for each element that shows the independence of goal from the alternatives. Simultaneously, $W_{33}$ is composed of a null matrix due to the independence of the alternatives amongst each other.

Table 1. Unweighted supermatrix with overall priority weights

\begin{tabular}{|c|c|c|c|c|c|c|c|c|c|c|c|c|}
\hline & GO & $\mathrm{EC}$ & $\mathrm{TL}$ & EN & SR & $\mathrm{A} 2 \mathrm{O}$ & $5 \mathrm{BP}$ & UCT & VIP & SBR & MBR & $\begin{array}{l}\text { overall } \\
\text { priority } \\
\text { weights }\end{array}$ \\
\hline $\mathrm{GO}$ & 1 & 1 & 1 & 1 & 1 & 1 & 1 & 1 & 1 & 1 & 1 & 1.00 \\
\hline $\mathrm{EC}$ & 0.22 & 0.56 & 0.24 & 0.40 & 0.58 & 0.27 & 0.40 & 0.26 & 0.28 & 0.20 & 0.31 & 0.32 \\
\hline TL & 0.25 & 0.16 & 0.58 & 0.27 & 0.14 & 0.28 & 0.24 & 0.23 & 0.27 & 0.24 & 0.17 & 0.26 \\
\hline EN & 0.33 & 0.11 & 0.09 & 0.21 & 0.09 & 0.17 & 0.16 & 0.30 & 0.27 & 0.29 & 0.28 & 0.23 \\
\hline SR & 0.21 & 0.17 & 0.09 & 0.12 & 0.19 & 0.27 & 0.19 & 0.21 & 0.18 & 0.27 & 0.24 & 0.19 \\
\hline $\mathrm{A} 2 \mathrm{O}$ & 0 & 0.39 & 0.29 & 0.05 & 0.10 & 0 & 0 & 0 & 0 & 0 & 0 & 0.24 \\
\hline $5 \mathrm{BP}$ & 0 & 0.11 & 0.23 & 0.16 & 0.05 & 0 & 0 & 0 & 0 & 0 & 0 & 0.14 \\
\hline UCT & 0 & 0.05 & 0.11 & 0.10 & 0.04 & 0 & 0 & 0 & 0 & 0 & 0 & 0.08 \\
\hline VIP & 0 & 0.08 & 0.09 & 0.13 & 0.05 & 0 & 0 & 0 & 0 & 0 & 0 & 0.09 \\
\hline SBR & 0 & 0.13 & 0.21 & 0.09 & 0.23 & 0 & 0 & 0 & 0 & 0 & 0 & 0.16 \\
\hline MBR & 0 & 0.24 & 0.06 & 0.46 & 0.53 & 0 & 0 & 0 & 0 & 0 & 0 & 0.3 \\
\hline
\end{tabular}

Presented in Fig.4 is the box-whisker plot of alternatives with their corresponding overall priority weights and variations. The most preferred BNR system to be installed in WTPs located in urban areas is the MBR system followed by A2O. In terms of economic aspect, MBR is now becoming a cheaper technology as it only requires lesser space. Moreover, different MBR configurations are studied in order to optimize and enhance the wastewater effluent quality [24]. The least preferred alternative is UCT which has a very close overall priority weight with VIP. Overall, the most important criterion is the economic aspect followed by technical aspect, environmental aspect and space requirement.

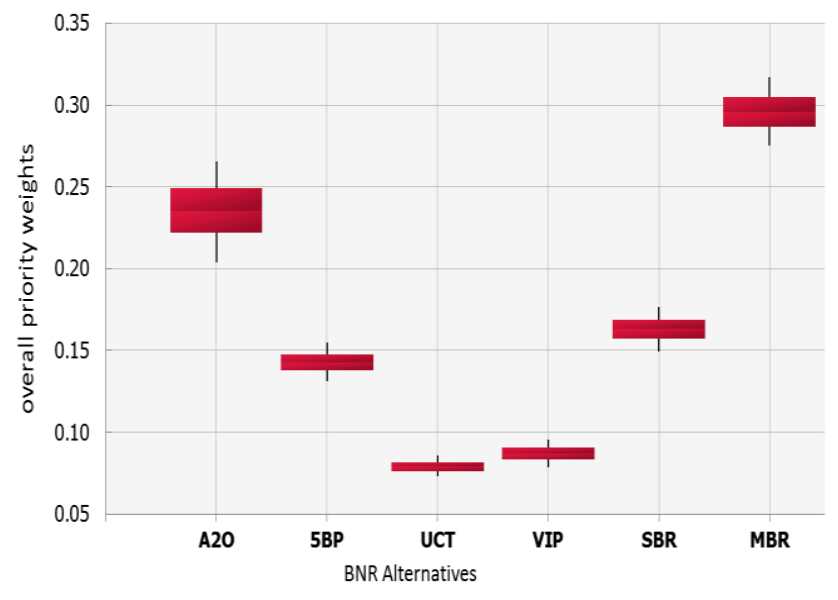

Fig. 4. Box-whisker plot of Monte Carlo simulation results

Furthermore, the uncertainties of the results are evaluated using the Monte Carlo simulation. An iteration of ten thousand times was adopted in order to come up with the distributions. The distributions were converted into a box-whisker plot as shown in Fig. 4. The box plot shows that the decision makers are consistent and certain that VIP and UCT are the least preferred BNR alternatives since the distributions for both alternatives show very low variations. SBR and 5BP, third and fourth in rank respectively, also have closer weights with overlapping whiskers. It can be observed that there is a relatively larger uncertainty and sensitivity in the resulting overall weight of $\mathrm{A} 2 \mathrm{O}$. However, the large variation and whisker length did not affect its rank. Though MBR has the second largest variation among the six alternatives, its rank as the best BNR system for WTPs in urban area is considered stable.

\section{Conclusion}

The study presents the complex decision making problem of selecting the best biological nutrient removal technology for wastewater treatment plants located in urban areas in a network structure. Fuzzy analytical network process and Monte Carlo simulations were utilized as tools to obtain the overall priority weights of the six alternatives considered. These tools could address the possible subjectivity and uncertainties in the evaluation of all the interacting elements in a systematic approach. The evaluation of the overall priorities shows that the membrane bioreactor is the best option followed by 3 -stage pho-redox and sequencing batch reactor. The most important criterion is the economic aspect then technical aspect. Upon utilizing Monte Carlo simulations, the variation for every alternative is shown with respect to the ranking. The results in this study could aid the stakeholders in addressing the decision making problem related to sustainable wastewater management. Future works would extend to considering sub-criteria for each criterion such as dividing economic aspect into upgrade or capital cost and operational cost. This would account a holistic evaluation of alternatives encompassing a more detailed network structure.

The authors would like to acknowledge the Philippine Council for Industry, Energy and Emerging Technology Research and Development (PCIEERD) under the Department of Science and Technology (DOST) for the support given to the Project NexCities. One of the authors, C.M. Pausta, is also supported by the Engineering Research and Development for Technology (ERDT) Program of DOST.

\section{References}

1. M. P. Chislock, E. Doster, R. A. Zitomer and A. E. Wilson, Nature Education Knowledge. 4, no. 4, 10 (2013)

2. R. Kleemann, J. Chenoweth, R. Clift, S. Morse, P. Pearce, and D. Saroj, Resour. Conserv. Recycl., 105, 347-359 (2015) 
3. E. B. Estrada-Arriaga et al., Sci. Total Environ., 571, 1172-1182 (2016)

4. The Cadmus Group, Nutrient control design manual: State of technology review report, 1-104 (2009)

5. Department of Environment and Natural Resources, Water Quality Guidelines and General Effluent Standards of 2016 (DENR Administrative Order 2016-08, Philippines, 2016)

6. M. A. Hamouda, W. B. Anderson, and P. M. Huck, Water Sci. Technol., 60, no. 7, 1767-1770 (2009)

7. G. A. Kiker, T. S. Bridges, A. Varghese, T. P. Seager, and I. Linkov, Integr. Environ. Assess. Manag., 1, (2), 95-108 (2005)

8. J. J. Wang, Y. Y. Jing, C. F. Zhang, and J. H. Zhao, Renew. Sustain. Energy Rev., 13, no. 9, 2263-2278 (2009)

9. T. L. Saaty, J. Math. Psychol., vol. 15, no. 3, 234 281 (1977)

10. T.L. Saaty, Decision Making with Dependence and Feedback: the Analytic Network Process, 2nd ed. (RWS Publica,ions, Pittsburgh, 2001)

11. C. M. Pausta, A. Huelgas-Orbecido, A. Beltran, R. C. Eusebio, J. J. Ignacio, and M. A. Promentilla, Selection of Optimum Biological Nutrient Removal ( BNR ) System for Urban Areas ' Wastewater Treatment Plants using Analytical Network Process ( ANP ), (2017)

12. M. A. B. Promentilla, J. F. D. Tapia, K. B. Aviso, and R. Raymond, 61, (2017)

13. M. A. B. Promentilla, T. Furuichi, K. Ishii, and N. Tanikawa, 62, (3), 308-324 (2006)

14. A. H. Orbecido, A. B. Beltran, R. A. J. Malenab, K. I. D. Miñano, M. A. B. Promentilla, Chem. Eng. Trans., 52, 643-648 (2016)

15. M.A. Promentilla, K. Aviso, R. Tan, Chem. Eng. Trans., 45, 829-834 (2015)

16. R. Tan, K. Aviso, A. Huelgas, M.A. Promentilla, Process Saf. Environ. Prot., 92, 467-475, (2014)
17. M. A. B. Promentilla, T. Furuichi, K. Ishii, and N. Tanikawa, Waste Manag., 26 (12), 1410-1421 (2006)

18. M. A. B. Promentilla, J. I. B. Janairo, and D. E. C. $\mathrm{Yu}, \mathbf{6 1},(2017)$

19. United States Environmental Protection Agency, Biological Nutrient Removal Processes and Costs, (Office of Water, Washington, 2007)

20. C. W. Randall and E. U. Cokgor, Performance and economics of BNR Plants in the Chesapeake Bay Watershed, USA, 21-28 (1995)

21. T. M. Gellner, Feasible and Cost Effective MBR Applications, 1-9 (2014)

22. L. R. Mayor, J. V. Camacho, and F. J. F. Morales, Water, Air, and Soil Pollution, 152, 279-296, (2004)

23. A. F. Silva et al., Appl. Microbiol. Biotechnol., 93, (5), 2171-2180 (2012)

24. M. Sibag and H. Kim, Bioresour. Technol., 104,173$180(2012)$ 